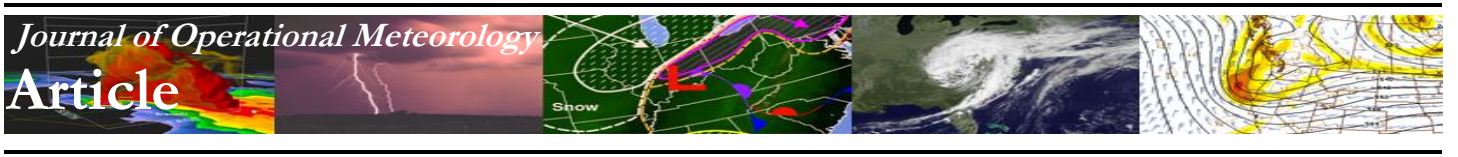

\title{
A Climatological Perspective on the 2011 Alabama Tornado Outbreak
}

\author{
PHILIP L. CHANEY \\ Auburn University, Auburn, Alabama \\ JONATHAN HERBERT and AMY CURTIS \\ Jacksonville State University, Jacksonville, Alabama
}

(Manuscript received 23 January 2012; in final form 17 September 2012)

\begin{abstract}
This paper presents a comparison of the recent 27 April 2011 tornado outbreak with a tornado climatology for the state of Alabama. The climatology for Alabama is based on tornadoes that affected the state during the 1981-2010 period. A county-level risk index is produced from this climatology. Tornado tracks from the 2011 outbreak are mapped and compared with the climatology and risk index. There were 62 tornadoes in Alabama on 27 April 2011, including many long-track and intense tornadoes. The event resulted in 248 deaths in the state. The 2011 outbreak is also compared with the April 1974 tornado outbreak in Alabama.
\end{abstract}

\section{Introduction}

Tornadoes have been documented in every state in the United States and on every continent except Antarctica. The United States has by far the most tornado reports annually of any country, averaging about 1,300 $\mathrm{yr}^{-1}$. Tornadoes form in thunderstorms, developing from the rotating updraft, or mesocyclone. Most form near fronts, particularly cold fronts and drylines associated with midlatitude cyclones (SPC 2011a).

Although not as well recognized as central parts of the country (i.e., the traditional "Tornado Alley"), the southeastern United States experiences many tornadoes (Bluestein 1999; Brooks et al. 2003). Activity takes place mostly during the spring, but with a secondary peak in the fall (Garinger and Knupp 1993). Recently it has been suggested that there is also a "Dixie Alley." Tornado path data do show that parts of Arkansas, Louisiana, Mississippi, and Alabama experience just as high a tornado risk as the traditional Tornado Alley region. This is especially true for strong tornadoes. There is little tornado activity in the Ouachita and Ozark Mountains, which may be due to complex terrain or lack of tornado reports due to low population density (Gagan et al. 2010; Dixon et al. 2011).

Alabama is affected in the spring and fall by midlatitude cyclones, often associated with severe weather and tornadoes. During summer and fall tornadoes also can be produced by tropical cyclones. A recent study suggests that most landfalling hurricanes on the Gulf Coast produce tornadoes, many of which have affected Alabama (Moore and Dixon 2011).

The overall number of tornadoes experienced every year in the United States has increased over the historical record (Boruff et al. 2003). This is most likely due to the increased observation and reporting of tornadoes over time, particularly F0 and F1 tornadoes. Over the 50-yr period from 1950 to 1999 deaths and injuries decreased and economic losses adjusted for inflation varied over time, peaking in the 1970s (Boruff et al. 2003).

However, in spite of continuing improvements in forecasts and warnings, certain areas of the country and populations are still more vulnerable than others. The reasons are most likely a combination of physical and social factors, including tornadoes occurring at night and in mountainous terrain, poverty, age, and low housing standards (Ashley 2007). Tornadoes can

Corresponding author address: Dr. Philip L. Chaney, 210 Petrie Hall, Department of Geology and Geography, Auburn University, Auburn, AL 36849

E-mail: chanepl@auburn.edu 
Table 1. Number of tornadoes in Alabama from 1981-2010, by F or EF scale. Percent indicates the percentage of all tornadoes per $\mathrm{F}$ or EF scale, and the 30-yr annual averages also are given.

\begin{tabular}{|c|c|c|c|}
\hline F or EF Scale & $\begin{array}{c}\text { Number of } \\
\text { tornadoes }\end{array}$ & Percent & $\begin{array}{c}\text { 30-yr Annual } \\
\text { Average }\end{array}$ \\
\hline 0 & 385 & 43 & 13 \\
\hline 1 & 341 & 38 & 11 \\
\hline 2 & 122 & 14 & 4 \\
\hline 3 & 41 & 4 & 1 \\
\hline 4 & 10 & 1 & $<1$ \\
\hline 5 & 1 & $<1$ & $<1$ \\
\hline Total & $\mathbf{9 0 0}$ & $\mathbf{1 0 0}$ & $\mathbf{3 0}$ \\
\hline
\end{tabular}

produce infrastructure damage that can affect individuals, businesses, and the local economy. Small communities in poorer areas are particularly affected, despite state or federal aid (Simmons and Sutter 2011).

The 2011 outbreak is the deadliest tornado outbreak on record, followed closely by the 3-4 April 1974 "Super Outbreak," which produced 148 tornadoes and 310 deaths. In the 1974 outbreak, there were seven F5-rated tornadoes compared to four EF5rated tornadoes in 2011 (SPC 2011a). The tornadoes in the 1974 outbreak affected similar areas of the country to those in 2011 (mainly focused in the southeastern states), although tornadoes occurred further north and in more Midwestern states in 1974 (Fujita 1975; Pearson and Ostby 1975). As in 2011, there were several F5-rated tornadoes and several with path lengths well over 100 miles (e.g., the tornadoes that hit Xenia, Ohio, and Guin, Alabama) (Fujita 1974).

Table 2. Same as Table 1 except for spring tornadoes. Spring is defined as March-May.

\begin{tabular}{|c|c|c|c|}
\hline F or EF Scale & $\begin{array}{c}\text { Number of } \\
\text { tornadoes }\end{array}$ & Percent & $\begin{array}{c}\text { 30-yr Annual } \\
\text { Average }\end{array}$ \\
\hline 0 & 147 & 38 & 5 \\
\hline 1 & 171 & 44 & 6 \\
\hline 2 & 44 & 11 & 1 \\
\hline 3 & 20 & 5 & $<1$ \\
\hline 4 & 5 & 1 & $<1$ \\
\hline 5 & 1 & $<1$ & $<1$ \\
\hline Total & $\mathbf{3 8 8}$ & $\mathbf{1 0 0}$ & $\mathbf{1 3}$ \\
\hline
\end{tabular}

The tornado outbreak of 25-28 April 2011 was of historic proportions. The severe weather and tornadoes from the event affected most of the eastern United States, especially the Southeast. On 27 April, the main day of the outbreak, there were 175 tornadoes and 316 deaths. Many of the tornadoes and the majority of deaths took place in Alabama (SPC 2011a,c).
This paper examines the tornado outbreak of 27 April 2011 in Alabama. Tornado track maps are produced from storm surveys and compared to the tornado climatology for the 30-yr period from 1981 to 2010. A county-level tornado risk index is produced from these data based on the number and strength of tornadoes historically affecting each county. Finally, the tornado tracks from the 2011 outbreak are compared with historic tracks and the risk index to see how unusual the event was from a climatological perspective.

\section{Data and methods}

In order to give some context to the 2011 outbreak in Alabama, a tornado climatology for the state is produced here. Historic tornado tracks from 1981-2010 were used for this analysis (SPC 2011b). Data are available back to 1950 in this database, but this time period was chosen for two main reasons. First, it uses the standard 30-yr period often used in climate analysis (e.g., for climate normals of elements such as temperature and precipitation). Second, it uses the latter half of the period of record, which should correct to some degree the problem of the increasing number of tornado reports over time.

There were a total of 900 tornadoes over the 1981-2010 period in Alabama, an average of $30 \mathrm{yr}^{-1}$. As expected, the majority of these were weak with only a small percentage of strong tornadoes. The number of tornadoes of each F- or EF-scale category is given in Table 1. The majority of tornadoes were weak, and frequency decreased with intensity. Eightyone percent of tornadoes were category 0 or category 1. Only about $2 \%$ were category 4 or 5 . Most tornadoes occurred in spring in Alabama. Seasons are defined here as winter (December-February), spring (March-May), summer (June-August), and fall (September-November). Table 2 shows the same data for the spring season alone. There were 388 tornadoes in spring, an average of $13 \mathrm{yr}^{-1}$. The intensity distribution was similar, except that there were more category 1 than 0 tornadoes compared with the annual data.

The historic tornado tracks for Alabama are shown in Figs. 1 and 2, for all seasons and for just spring, respectively. In both cases, most tornadoes exhibited a southwest to northeast track, mimicking thunderstorm movement driven by synoptic-scale circulation in 


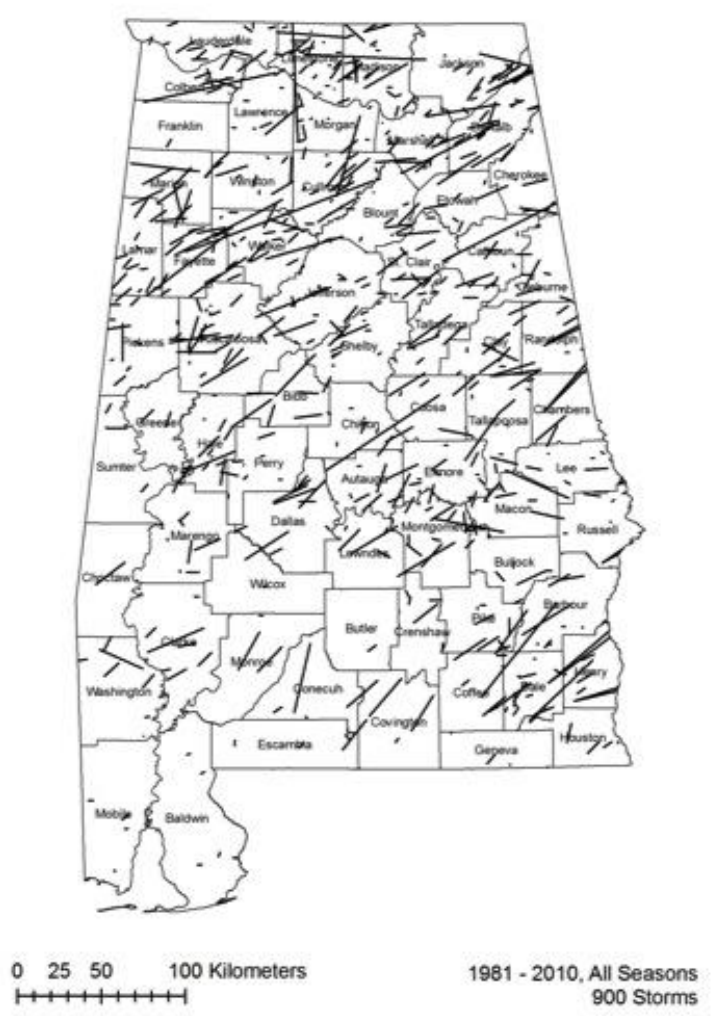

Figure 1. All tornado tracks in Alabama during 1981-2010. Click image for an external version; this applies to all figures hereafter.

midlatitude cyclones. Another generalization is that there were more tornadoes in northern versus southern Alabama. Again, this pattern suggests a synoptic-scale effect, associated with the position of air masses and cold fronts.

The tracks from the 2011 outbreak are shown in Fig. 3. These were digitized using the start and end locations in latitude and longitude from individual tornado surveys carried out by local National Weather Service offices in Birmingham, Huntsville, and Mobile (NWS 2011). One pattern that is immediately apparent is the sheer number of tornadoes and the length of the tracks that affected central and northern Alabama.

The historic data also were summarized by county. A county was considered impacted by a tornado if the track intercepted the county boundary. Thus, a single tornado could be counted in several counties. The county-level data were used to construct a tornado risk index. The index takes into account the number of tornadoes and their strength on the F or EF scale. For each county, the number of tornadoes of each category was multiplied by the minimum wind speed strength of that category on the EF scale. In order from EF0 to EF5, these are 105, 138, 179, 219, 267, and $322 \mathrm{~km} \mathrm{~h}^{-1}$
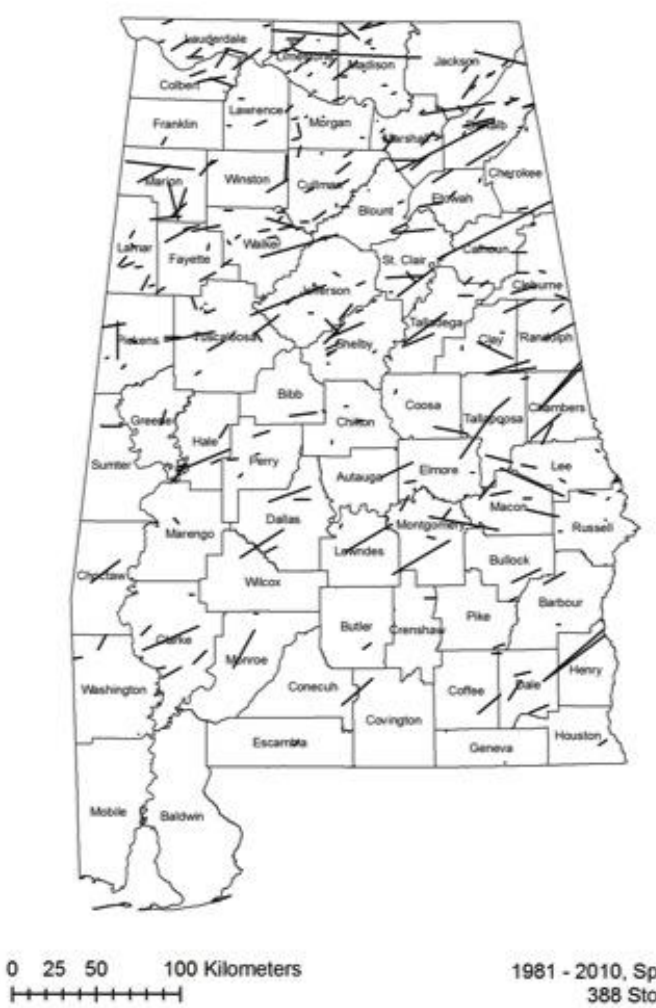

1981 - 2010 . Spring
388 Storms

Figure 2. Same as Fig. 1 except for spring (March-May) tornado tracks.
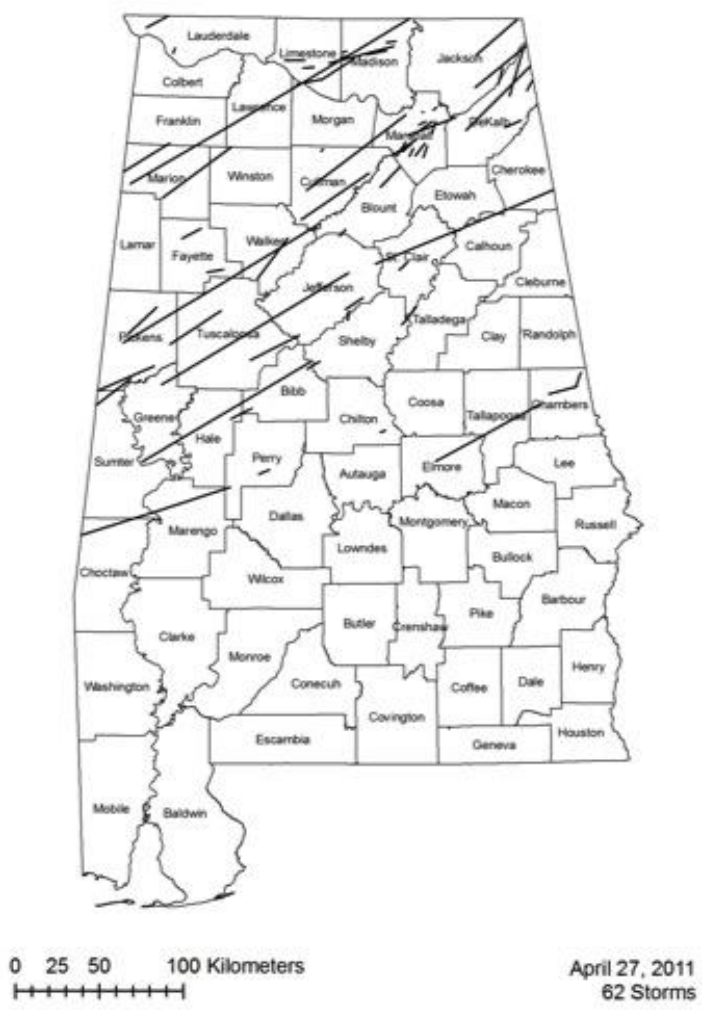

Figure 3. Tornado tracks in Alabama on 27 April 2011. 
$\left(65,86,111,136,166\right.$, and $\left.200 \mathrm{mi} \mathrm{h}^{-1}\right)$ respectively. These numbers were then summed and divided by the area of each county to produce the risk index. For each county, the index was constructed as follows:

Index $=\sum_{i=0}^{5} \frac{x_{i} y_{i}}{a}$

Where $x$ is the number of tornadoes, $y$ is the minimum wind speed on the EF scale for a given category $(0-5)$, and $a$ is the county area. This also can be written as follows:

Index $=\left[\left(x_{0} \times 105\right)+\left(x_{1} \times 138\right)+\left(x_{2} \times 179\right)\right.$

$\left.+\left(x_{3} \times 219\right)+\left(x_{4} \times 267\right)+\left(x_{5} \times 322\right)\right] / a$

The index was classified using a Natural Breaks technique (Dent et al. 2008) into low, medium, and high categories. Although wind speeds are slightly different on the F and EF scales, the newer scale was used here for all tornadoes since the tornadoes in the database were classified on this scale. The index is shown in Fig. 4. The index is highest in a corridor stretching from Tuscaloosa County in central Alabama to Limestone, Madison, Jackson, and DeKalb Counties in northeastern Alabama. Northern and southeastern Alabama have a medium risk, and the lowest risk is found in southwestern parts of the state.

\section{Results and discussion}

\section{a. 2011 tornado outbreak}

The 2011 outbreak took place over several days, beginning on 25 April and ending on 28 April. Most of the severe weather was concentrated on one day, 27 April, on which there were 62 tornadoes in Alabama. As in 1974, the outbreak was produced by a midlatitude cyclone. The warm, humid conditions ahead of its cold front, combined with an upper-level trough and strong jet stream, provided the instability and wind shear for tornadic thunderstorms. As the cold front pushed eastward during the day, it produced two waves of severe storms. The first wave, between 0400 and 0700 am CDT (0900 and 1200 UTC), was associated with a squall line that produced 29 tornadoes. The second wave, between 1100 am and $1000 \mathrm{pm}$ CDT (1600 and 0300 UTC), was associated with an explosion of long-lived supercell storms, which produced 33 tornadoes, including all the day's EF4- and EF5-rated tornadoes (SPC 2011a).

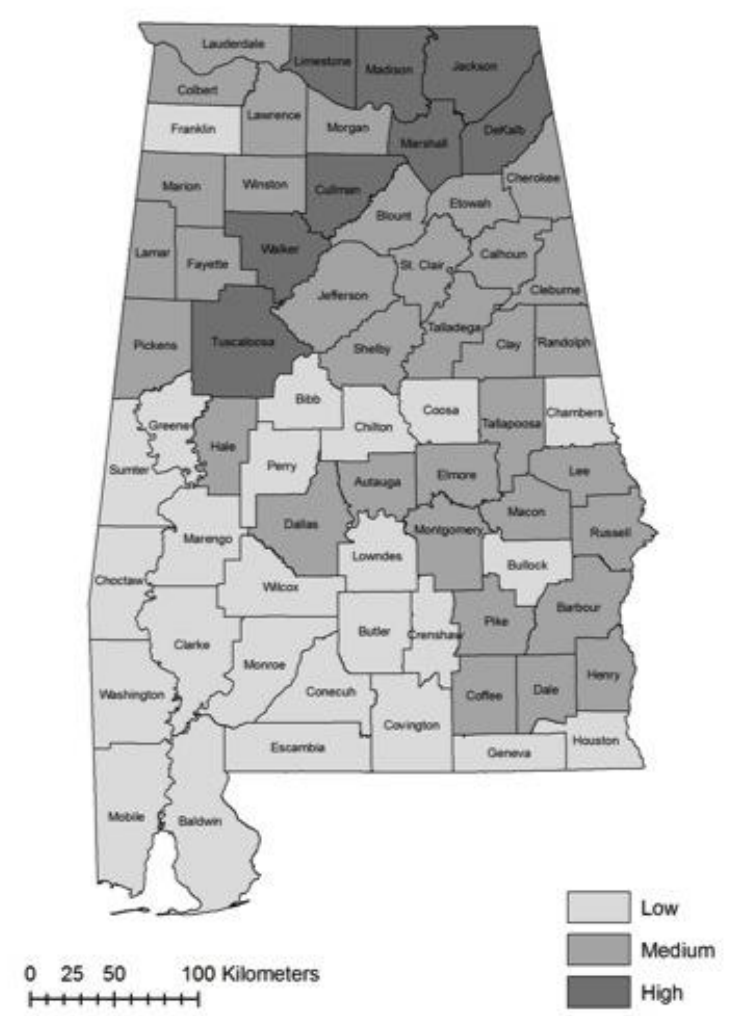

Figure 4. Tornado risk index. The index is based on the number and strength of tornadoes that hit each county in the 1981-2010 time period per equations (1) or (2).

The number of tornadoes in the outbreak of each EF scale is given in Table 3. The number of deaths in Alabama attributed to the outbreak stands at 235 according to SPC (2011c). The Alabama Emergency Management Agency has a slightly higher number of 248, which includes indirect fatalities such as those that occurred after 27 April but were related to injuries sustained during the event. The number of deaths by county is given in Table 4 (Alabama Emergency Management Agency 2011).

Table 3. Number of tornadoes in Alabama on 27 April 2011.

\begin{tabular}{|c|c|c|}
\hline EF Scale & $\begin{array}{c}\text { Number of } \\
\text { tornadoes }\end{array}$ & Percent \\
\hline 0 & 6 & 10 \\
\hline 1 & 30 & 48 \\
\hline 2 & 8 & 13 \\
\hline 3 & 9 & 15 \\
\hline 4 & 7 & 11 \\
\hline 5 & 2 & 3 \\
\hline Total & $\mathbf{6 2}$ & $\mathbf{1 0 0}$ \\
\hline
\end{tabular}

The outbreak produced two EF5-rated tornadoes. The first EF5-rated tornado was the longest track 
Table 4. Tornado-related fatalities in Alabama resulting from the 27 April 2011 tornado outbreak, by county (Alabama Emergency Management Agency 2011).

\begin{tabular}{|c|c|c|c|}
\hline County & Fatalities & County & Fatalities \\
\hline Bibb & 1 & Lawrence & 14 \\
\hline Calhoun & 9 & Limestone & 4 \\
\hline Cullman & 2 & Madison & 9 \\
\hline Dekalb & 35 & Marion & 25 \\
\hline Elmore & 6 & Marshall & 5 \\
\hline Fayette & 4 & St. Clair & 15 \\
\hline Franklin & 26 & Tallapoosa & 1 \\
\hline Hale & 6 & Tuscaloosa & 48 \\
\hline Jackson & 8 & Walker & 9 \\
\hline Jefferson & 21 & Total & $\mathbf{2 4 8}$ \\
\hline
\end{tabular}

tornado of the day, approximately $210 \mathrm{~km}(130 \mathrm{mi})$ in Alabama. It affected Marion, Franklin, Lawrence, Limestone, and Madison Counties and did damage to the communities of Hackleburg, Phil Campbell, Oak Grove, and Mount Hope. It touched down at 0305 pm CDT (2005 UTC) and was on the ground for over two hours until lifting off the ground at $0520 \mathrm{pm}$ CDT (2220 UTC) in Franklin County, Tennessee.

The second EF5-rated tornado was the approximately 50-km (30 mi) track tornado in DeKalb County that caused damage in Rainsville and Sylvania. It touched down at 0619 pm CDT (2319 UTC) and was on the ground for approximately 37 minutes before lifting off the ground at 0656 pm CDT (2356 UTC) in Dade County, Georgia. There were several other long-track tornadoes in the outbreak and several with paths approximately $1.6 \mathrm{~km}(1.0 \mathrm{mi})$ wide, including the EF5-rated tornadoes mentioned here and the highly publicized tornado that went through the cities of Tuscaloosa and Birmingham. This tornado was classified as an EF4 but accounted for most of the fatalities in the state.

The tracks from the outbreak are superimposed on the risk index in Fig. 5. Many of the tornadoes from the outbreak occurred in areas with a high tornado risk index. Many tornadoes also occurred in medium risk areas in northern parts of the state. The event fits fairly well with the index. When comparing tracks from this event to the historic tornado tracks for spring (Fig. 2), however, it was clearly unusual. Most spring tornadoes were historically short track tornadoes and affected almost every part of the state. The recent outbreak's tornadoes were numerous, long-track tornadoes, affecting mostly the northern part of the state.

The strength/intensity of the tornadoes from the 2011 outbreak also was unusual. There was a lower percentage of EF0-rated tornadoes than the spring average and a higher percentage of every other

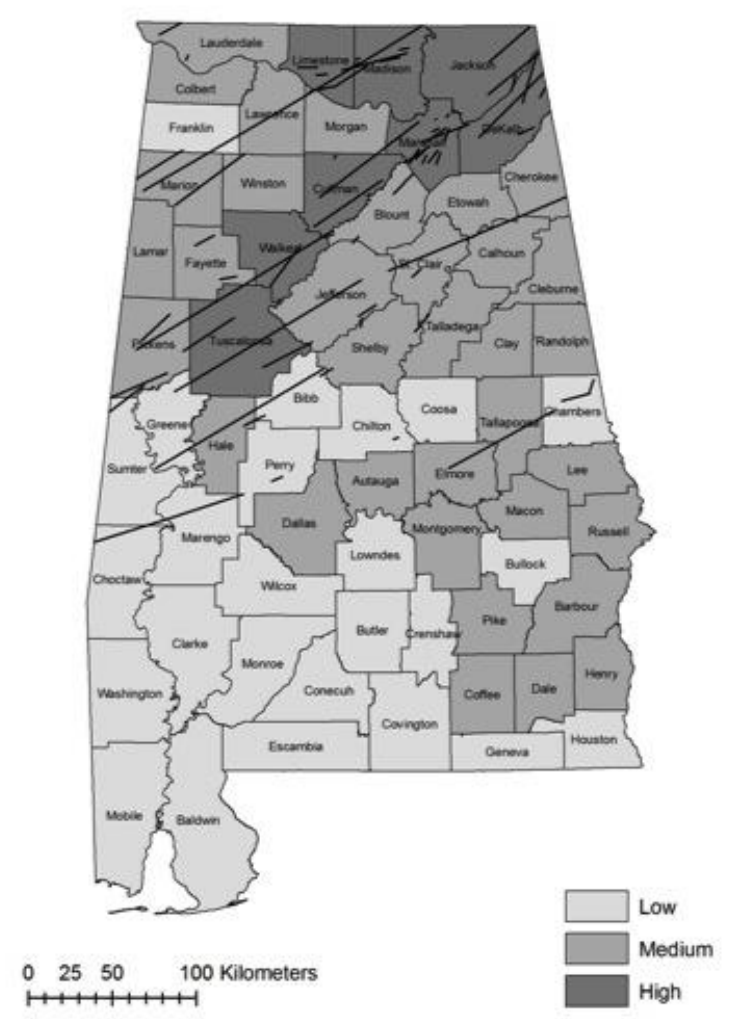

Figure 5. Tornado risk index (1981-2010) as in Fig. 4 along with tornado tracks from 27 April 2011.
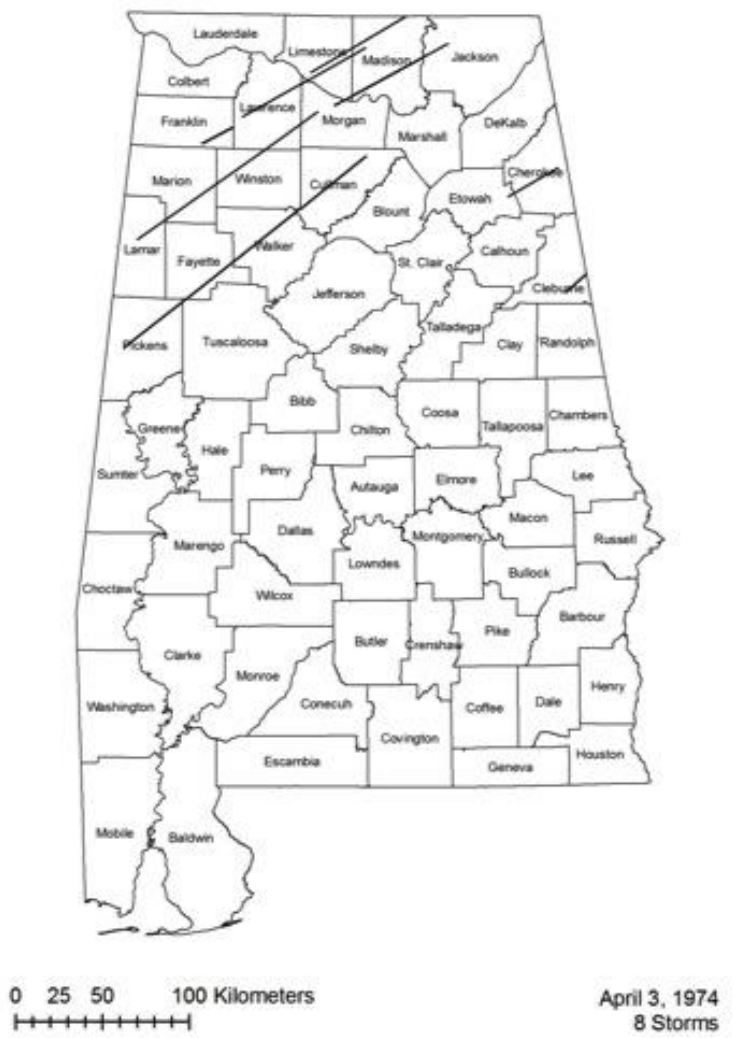

Figure 6. Tornado tracks in Alabama on 3 April 1974. 
category. Most notably, $42 \%$ of the tornadoes were rated strong (EF2 to EF3) or violent (EF4 to EF5), compared to $18 \%$ on average. In terms of sheer numbers of tornadoes, there were 62 tornadoes in one day compared to an average of 13 in a typical spring tornado season.

\section{b. 1974 tornado outbreak}

The 1974 outbreak was produced by similar meteorological conditions to the 2011 outbreak. A series of three or four convective bands produced thunderstorm and tornado activity in the warm sector of a midlatitude cyclone. The activity was triggered by particularly warm, humid conditions at the surface, an advancing cold front, and strong jet stream winds in an upper-level trough. Other factors such as a short-wave trough and dryline may have also contributed to some of the lifting (Corfidi et al. 2010).

The tracks from the 1974 tornado outbreak that affected Alabama are shown in Fig. 6. Eight tornado tracks in the Storm Prediction Center database are used here. Comparing the 1974 and 2011 outbreaks, there are similarities and differences. The tornadoes officially reported in the 1974 outbreak were longtrack tornadoes that affected counties in northern Alabama, including many of the same counties later affected in the 2011 outbreak. All of the counties where people died in the 1974 outbreak also recorded deaths in the 2011 outbreak, with the exception of Winston County.

The main difference was the number of tornadoes recorded in Alabama during the outbreaks, 8 in 1974 versus 62 in 2011. Even though there were doubtless many smaller tornadoes that went unrecorded in 1974 due to underreporting or lack of Doppler radar, the recent outbreak clearly involved more tornadoes and affected a larger portion of the state.

\section{Conclusions}

The climatology and risk index presented here help to put the recent tornado outbreak of 2011 in Alabama in context. The tornadoes of 27 April 2011 did occur in areas of the state that have historically observed tornadoes. It was the sheer number of tornadoes - twice the number of tornadoes in one day as would be typically expected in one year-as well as their intensity and length of time on the ground, that likely contributed to the large death toll. The tornado paths from the recent outbreak and the destruction left behind were still easily seen several months later in many parts of the state.

The topography of Alabama can be generalized as mountainous in the north and coastal plain in the south. The Appalachian Mountains are found in the northeastern parts of the state, dominated by ridge and valley terrain, changing to plateau in the northwest. Interestingly, many of the tornadoes tracked over areas of this complex topography, particularly along Sand and Lookout Mountains in northeastern Alabama, part of the Cumberland Plateau.

Population may affect the construction of indices like the risk index presented here. Where there are more people we may expect more tornadoes to be reported, producing a population bias. In this particular case the index is high in many counties with larger populations such as Tuscaloosa County (population 194,656) and Madison County (population 334,811), which include the cities of Tuscaloosa and Huntsville (United States Census Bureau 2011). However, the index was successful in identifying the appropriate risk level in areas where population bias might be expected to severely distort the results. For example, Jefferson County, with the largest population in the state $(658,466)$, is classified as a medium-risk area while many counties with low populations, such as DeKalb County (population 71,109), are classified as high-risk areas.

Cutter (1996) discussed the vulnerability of a location as a function of both physical and social factors. The tornado risk index developed for Alabama in this study is based solely on physical factors. In order to more fully understand the vulnerability of any particular place in Alabama to tornadoes, the social vulnerability characteristics of the place must be considered as well. Cutter et al. (2003) developed a social vulnerability index at the county level for the United States that could be used for this purpose; however, a social vulnerability index tailored specifically for tornadoes might be more appropriate (Chaney and Weaver 2010). Either way, this task is beyond the scope of the research presented herein.

Acknowledgements. The authors would like to thank the staff at the National Weather Service office in Birmingham, $\mathrm{AL}$, for their help and for keeping us up to date with tornado damage survey information across the state after the tornado outbreak. We also thank Mr. John Gagan and Dr. Sam Ng for their insightful comments on the manuscript. 


\section{REFERENCES}

Alabama Emergency Management Agency, cited 2011: Alabama tornado related deaths, press release October 3, 2011. [Available online at ema.alabama.gov.]

Ashley, W., S., 2007: Spatial and temporal analysis of tornado fatalities in the United States: 1880-2005. Wea. Forecasting, 22, 1214-1228.

Bluestein, H. B., 1999: Tornado Alley: Monster Storms of the Great Plains. Oxford University Press, 180 pp.

Boruff, B. J., J. A. Easoz, S. D. Jones, H. R. Landry, J. D. Mitchem, and S. L. Cutter, 2003: Tornado hazards in the United States. Climate Res., 24, 103-117.

Brooks, H. E., C. A. Doswell III, and M. P. Kay, 2003: Climatological estimates of local daily tornado probability for the United States. Wea. Forecasting, 18, 626-640.

Chaney, P. L., and G. S. Weaver, 2010: The vulnerability of mobile home residents in tornado disasters: The 2008 Super Tuesday tornado in Macon County, Tennessee. Wea. Climate Soc., 2, 190-199.

Corfidi, S., S. Weiss, J. Cain, S. Corfidi, R. Rabin, and J. Levit, 2010: Revisiting the 3-4 April 1974 super outbreak of tornadoes. Wea. Forecasting, 25, 465-510.

Cutter, S. L., 1996: Vulnerability to environmental hazards. Prog. Hum. Geogr., 20 (4), 529-539.

, B. J. Boruff, and W. L. Shirley, 2003: Social vulnerability to environmental hazards. Soc. Sci. Quart., 84 (2), 242-261.

Dent, B. D., J. S. Torguson, and T. W. Hodler, 2008: Cartography: Thematic Map Design. McGraw-Hill, $368 \mathrm{pp}$.

Dixon, P. G., A. E. Mercer, J. Choi, and J. S. Allen, 2011: Tornado risk analysis: Is Dixie Alley an extension of Tornado Alley? Bull. Amer. Meteor. Soc., 92, 433-441.

Fujita, T. T., 1974: Jumbo tornado outbreak of 3 April 1974. Weatherwise, 27 (3), 116-126.
1975: Color map of superoutbreak tornadoes of 3-4 April 1974. Weatherwise, 28 (2), 54-55.

Gagan, J. P., A. Gerard, and J. Gordon, 2010: A historical and statistical comparison of "Tornado Alley" to "Dixie Alley". Natl. Wea. Dig., 34 (2), 145-155.

Garinger, L. P., and K. R. Knupp, 1993: Seasonal tornado climatology for the Southeastern United States. The Tornado: Its Structure, Dynamics, Prediction, and Hazards, Geophys. Monogr., 79, Amer. Geophys. Union, 445-452.

Moore, T. W., and R. W. Dixon, 2011: Climatology of tornadoes associated with Gulf Coast landfalling hurricanes. Geographical Review, 101 (3), 371-395.

NWS, cited 2011: Historic tornado outbreak April 27, 2011. National Weather Service, Birmingham, AL. [Available online at www.srh.noaa.gov/bmx/?n=event_04272011.]

Pearson, A., and F. P. Ostby Jr., 1975: The tornado season of 1974. Weatherwise, 28 (1), 4-12.

Simmons, K. M., and D. Sutter, 2011: Economic and Societal Impacts of Tornadoes. University of Chicago Press, $282 \mathrm{pp}$.

SPC, cited 2011a: Frequently asked questions about tornadoes. Storm Prediction Center, Norman, OK. [Available online at www.spc.noaa.gov/faq/tornado/ \#History.]

SPC, cited 2011b: SVRGIS. Storm Prediction Center, Norman, OK. [Available online www.spc.noaa.gov/gis/ svrgis.]

SPC, cited 2011c: Annual U.S. killer tornado statistics. [Available online at http://www.spc.noaa.gov/climo/ torn/fataltorn.html.]

United States Census Bureau, cited 2011: State \& county quickfacts. [Available online at quickfacts.census.gov/ qfd/states/01000.html.] 